\title{
PET Mapping for Brain-Computer Interface Stimulation of the Ventroposterior Medial Nucleus of the Thalamus in Rats with Implanted Electrodes
}

\author{
Yunqi Zhu*1-5, Kedi Xu*6, Caiyun $\mathrm{Xu}^{* 1-5}$, Jiacheng Zhang ${ }^{6}$, Jianfeng $\mathrm{Ji}^{1-5}$, Xiaoxiang Zheng ${ }^{6}$, Hong Zhang ${ }^{1-5}$, \\ and Mei Tian ${ }^{1-5}$ \\ ${ }^{I}$ Department of Nuclear Medicine, Second Hospital of Zhejiang University School of Medicine, Hangzhou, China; ${ }^{2}$ Zhejiang \\ University Medical PET Center, Hangzhou, China; ${ }^{3}$ Key Laboratory of Medical Molecular Imaging of Zhejiang Province, Hangzhou, \\ China; ${ }^{4}$ Institute of Nuclear Medicine and Molecular Imaging, Zhejiang University, Hangzhou, China; ${ }^{5}$ Collaborative Innovation \\ Center for Brain Science, Fudan University, Shanghai, China; and ${ }^{6}$ Qiushi Academy for Advanced Studies (QAAS), Zhejiang \\ University, Hangzhou, China
}

Brain-computer interface $(\mathrm{BCl})$ technology has great potential for improving the quality of life for neurologic patients. This study aimed to use PET mapping for $\mathrm{BCl}$-based stimulation in a rat model with electrodes implanted in the ventroposterior medial (VPM) nucleus of the thalamus. Methods: PET imaging studies were conducted before and after stimulation of the right VPM. Results: Stimulation induced significant orienting performance. ${ }^{18}$ F-FDG uptake increased significantly in the paraventricular thalamic nucleus, septohippocampal nucleus, olfactory bulb, left crus II of the ansiform lobule of the cerebellum, and bilaterally in the lateral septum, amygdala, piriform cortex, endopiriform nucleus, and insular cortex, but it decreased in the right secondary visual cortex, right simple lobule of the cerebellum, and bilaterally in the somatosensory cortex. Conclusion: This study demonstrated that PET mapping after VPM stimulation can identify specific brain regions associated with orienting performance. PET molecular imaging may be an important approach for $\mathrm{BCl}-$ based research and its clinical applications.

Key Words: positron emission tomography (PET); brain-computer interface (BCl); electrical stimulation; ventroposterior medial (VPM) nucleus of the thalamus

J Nucl Med 2016; 57:1141-1145

DOI: 10.2967/jnumed.115.171868

\section{B} rain-computer interface (BCI) technology has gained great visibility in the past few years as it merges the fields of biorobotics and neuroscience. Clinically, it is a promising therapeutic strategy for the restoration of sensory and motor function in patients with neurologic disorders $(1,2)$. Because of the technical advancement of implantable microelectrodes and processing electronics,

\footnotetext{
Received Dec. 28, 2015; revision accepted Feb. 10, 2016.

For correspondence or reprints contact: Mei Tian, Department of Nuclear Medicine, Second Affiliated Hospital of Zhejiang University School of Medicine, 88 Jiefang Rd., Hangzhou, Zhejiang 310009, China.

E-mail: meitian@zju.edu.cn

${ }^{*}$ Contributed equally to this work.

Published online Feb. 25, 2016.

COPYRIGHT (c) 2016 by the Society of Nuclear Medicine and Molecular Imaging, Inc.
}

the initial achievement of remote control of an animal's orienting performance has been succeeded by repeated training during artificial introduction of electrical commands into the somatosensory cortical and medial forebrain bundles (3). During the training, rats learn to interpret remote brain stimulation as instructions directing their trajectory of locomotion. Recently, our group demonstrated a novel control method by which direct stimulation of the ventroposterior medial (VPM) nucleus of the thalamus can initiate orienting performance in freely roaming rats without repeated training sessions. Supplemental Video 1 shows an example of this BCI-guided rat navigation. In the directions indicated by the arrows, the rat in the video was instructed to climb a slope, cross a bridge, and turn left or right (4). VPM is known as a somatosensory relay station that takes sensory information being input from individual whiskers and projects it to the primary somatosensory cortex (5). However, it is unclear which brain regions are involved in the orienting performance induced by VPM stimulation. Thus, we hypothesized that by using a PET molecular imaging approach to map the brain, we could explore the VPM stimulation-related orienting function and identify the specific cerebral activation pattern. In the present study, we conducted PET imaging on a freely moving rat model before and after VPM stimulation. To our knowledge, this was the first PET imaging study on BCIbased stimulation in a rat model with electrodes implanted in the VPM.

\section{MATERIALS AND METHODS}

\section{Animals and Electrode Implantation}

Twelve male Sprague-Dawley rats (weight range, 250-280 g) were used for this study. Bipolar electrical-stimulation electrodes were constructed with insulated nickel-chromium resistance alloy wire (Nichrome; bar diameter, $50 \mu \mathrm{m}$; $0.4 \mathrm{~mm}$ between electrode tips [A-M Systems]) (4). The animals were secured to a stereotactic frame (RWD Life Science Co.) under pentobarbital sodium anesthesia $(50 \mathrm{mg} / \mathrm{kg}$, intraperitoneally). The electrodes were then implanted into the right VPM according to a rat brain atlas (6) and affixed to the skull by dental cement. The animals were allowed to recover for $1 \mathrm{wk}$ before we began the experiments, which were approved by the Institutional Animal Care and Use Committee at Zhejiang University. 


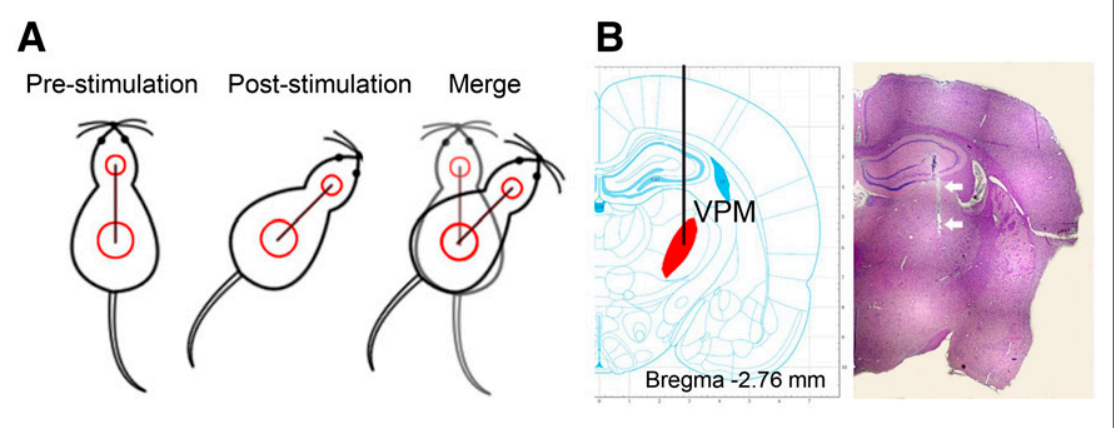

FIGURE 1. (A) Illustration of how turning angle is calculated from difference in position direction before and after stimulation. (B) Hematoxylin and eosin staining of tissue from electrode site, with electrode track indicated by arrows. were performed on a small-animal microPET R4 scanner (Siemens Medical Solutions) 7 and $10 \mathrm{~d}$, respectively, after the stereotactic surgery. In the poststimulation studies, the rats were subjected to VPM stimulation for 30 min immediately after receiving an injection of ${ }^{18} \mathrm{~F}$-FDG $(18.5 \mathrm{MBq})$ via the tail vein. The PET images were acquired $40 \mathrm{~min}$ after ${ }^{18} \mathrm{~F}$-FDG injection and analyzed using an improved toolbox for voxelwise analysis of rat brain images available in SPM, version 8.0 (Wellcome Trust Centre for Neuroimaging) (7). Groups were compared using a paired $t$ test with a significance threshold of $P<0.001$.

\section{Stimulation and Orienting Performance}

To deliver electrical stimulation directly into the VPM while allowing the animal to move freely, a flexible extension cable was used to connect an isolated stimulator (A-M Systems) to the implanted electrodes. The stimulus paradigm was a 30-min block of 180 cycles consisting of $1 \mathrm{~s}$ of stimulation followed by $9 \mathrm{~s}$ of rest. The stimulationinduced orienting performance was captured by a video camera, and the turning angle was calculated as shown in Figure 1A. A turningangle range of $30^{\circ}-60^{\circ}$ was regarded as indicating a successful stimulus. Animals whose turning angle was less than $30^{\circ}$ or larger than $60^{\circ}$ were excluded from the study.

\section{PET Protocol and Data Analysis}

All animals were deprived of food overnight before undergoing the PET imaging studies. Baseline and poststimulation PET studies

\section{RESULTS}

On completion of poststimulation PET imaging, the rats were sacrificed, the brains excised, and sections stained with hematoxylin and eosin. Pathologic examination confirmed that the electrodes had been implanted at the appropriate site. A representative hematoxylin- and eosin-stained image is presented in Figure 1B.

The stimulation induced ipsilateral orienting performance toward the right side, as shown in Supplemental Video 2. Among the 12 tested rats, 8 showed an appropriate turning angle of $30^{\circ}-60^{\circ}$ during stimulation; the other 4 had turning angles of less than $30^{\circ}$ and thus were excluded from the study.

After stimulation, ${ }^{18}$ F-FDG uptake was significantly increased in the paraventricular thalamic nucleus, septohippocampal

TABLE 1

Significant Metabolic Changes after VPM Stimulation (Baseline vs. Stimulation)

\begin{tabular}{|c|c|c|c|c|c|c|}
\hline \multirow[b]{2}{*}{ Region } & \multicolumn{3}{|c|}{ Coordinate (mm) } & \multicolumn{3}{|c|}{ Cluster level } \\
\hline & $x$ & $y$ & $z$ & $t$ value & $z$ score & Uncorrected $P$ \\
\hline Paraventricular thalamic nucleus & 0 & 5 & -2 & 30.02 & 5.70 & $<0.001$ \\
\hline Septohippocampal nucleus & 0 & 4 & 0 & 30.02 & 5.70 & $<0.001$ \\
\hline Right lateral septum & 1 & 5 & 0 & 30.02 & 5.70 & $<0.001$ \\
\hline Left lateral septum & -1 & 5 & 0 & 30.02 & 5.70 & $<0.001$ \\
\hline Right amygdala & 5 & 8 & -3 & 11.16 & 4.41 & $<0.001$ \\
\hline Right piriform cortex & 6 & 9 & -3 & 11.16 & 4.41 & $<0.001$ \\
\hline Right endopiriform nucleus & 6 & 8 & -2 & 5.75 & 3.39 & $<0.001$ \\
\hline Right insular cortex & 6 & 8 & -2 & 5.75 & 3.39 & $<0.001$ \\
\hline Left amygdala & -5 & 8 & -3 & 6.86 & 3.67 & $<0.001$ \\
\hline Left piriform cortex & -6 & 9 & -3 & 5.41 & 3.29 & $<0.001$ \\
\hline Left endopiriform nucleus & -6 & 8 & -2 & 6.86 & 3.67 & $<0.001$ \\
\hline Left insular cortex & -6 & 8 & -2 & 6.86 & 3.67 & $<0.001$ \\
\hline Olfactory bulb & 0 & 3 & 6 & 7.42 & 3.80 & $<0.001$ \\
\hline Left crus II of ansiform lobule of cerebellum & -4 & 5 & -13 & 6.76 & 3.65 & $<0.001$ \\
\hline Right primary somatosensory cortex & 5 & 2 & 0 & 9.17 & 4.12 & $<0.001$ \\
\hline Left primary somatosensory cortex & -5 & 1 & 3 & 8.13 & 3.94 & $<0.001$ \\
\hline Right secondary visual cortex & 5 & 3 & -9 & 6.30 & 3.54 & $<0.001$ \\
\hline Right simple lobule of cerebellum & 3 & 1 & -10 & 5.13 & 3.20 & $<0.001$ \\
\hline
\end{tabular}




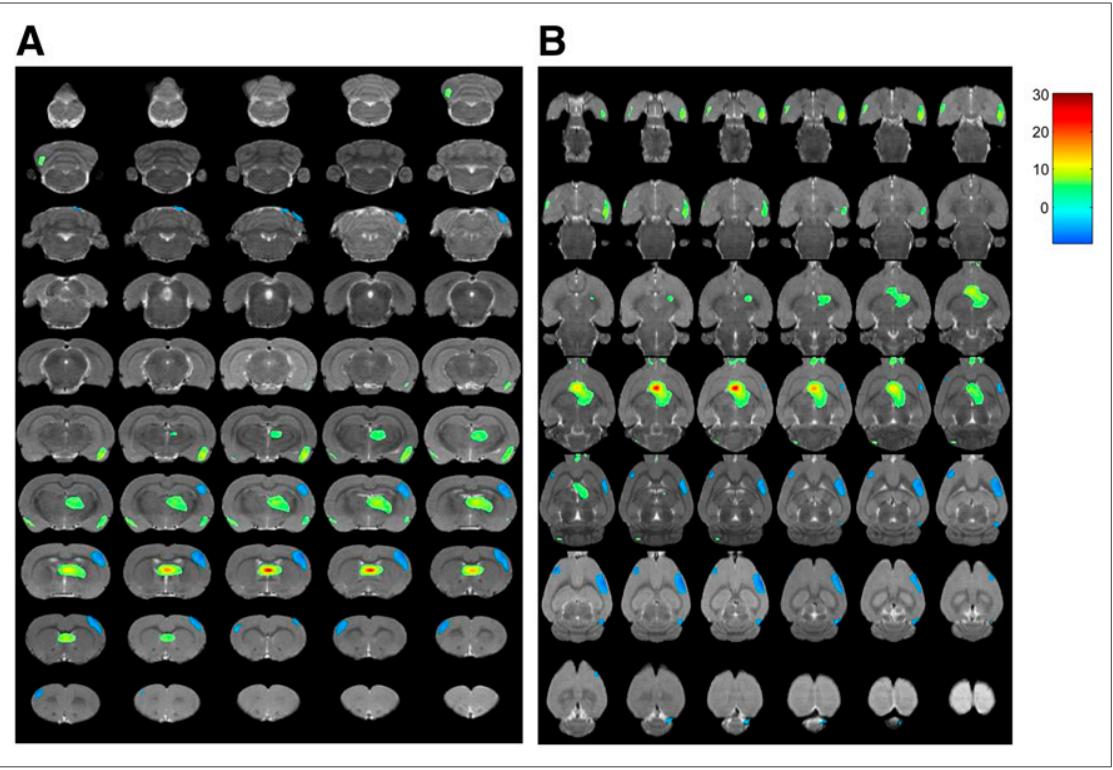

FIGURE 2. In vivo PET images of rat brain. Serial coronal (A) and transverse (B) sections demonstrate significant changes in glucose metabolism after VPM stimulation $(P<0.001)$. Differences in brain regions are color-coded and superimposed on MRI template.

nucleus, olfactory bulb, left crus II of the ansiform lobule of the cerebellum, and bilaterally in the lateral septum, amygdala, piriform cortex, endopiriform nucleus, and insular cortex (Table 1; Figs. 2A and 3) but was significantly decreased in the right secondary visual cortex, right simple lobule of the cerebellum, and bilaterally in the primary somatosensory cortex (Table 1; Figs. 2B and 4).

\section{DISCUSSION}

Born as a highly multidisciplinary field, BCI research has moved at a stunning pace since the first experiment demonstrating

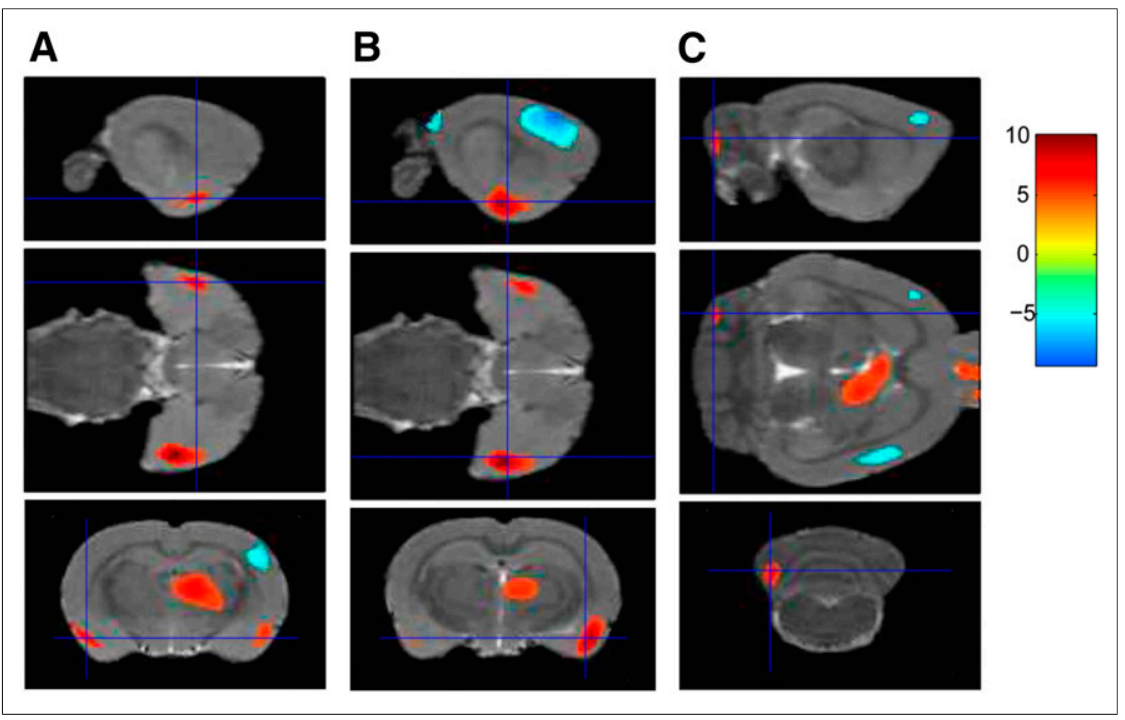

FIGURE 3. Representative sagittal (top), transverse (middle), and coronal (bottom) PET images demonstrating increased glucose metabolism in left amygdala (A), right amygdala (B), and left crus II (C) $(P<0.001)$ after VPM stimulation. that electrical activity generated by ensembles of cortical neurons can directly control a robotic manipulator $(8)$. Noninvasive neuroimaging modalities for BCI systems are gaining momentum in the research arena. Although functional MRI, with its superior spatial resolution, has surfaced as the major tool for noninvasive characterization of brain function (9), PET has the advantage of tracing biochemical changes in cognitive or behavioral brain function during real-time tasks without the interference of ambient noise generated by the MRI scanner, and unlike MRI, PET is suitable for a brain with implanted metal electrodes.

In the current study, by using ${ }^{18} \mathrm{~F}-\mathrm{FDG}$ PET imaging we found that VPM stimulation induced an increase in glucose metabolism in the paraventricular thalamic nucleus, septohippocampal nucleus, olfactory bulb, left crus II, and bilaterally in the lateral septum, amygdala, piriform cortex, endopiriform nucleus, and insular cortex but that a significantly decrease in

${ }^{18} \mathrm{~F}-\mathrm{FDG}$ accumulation occurred in the right secondary visual cortex, right simple lobule of the cerebellum, and bilaterally in the primary somatosensory cortex. To the best of our knowledge, this was the first study using PET as a neuroimaging tool to investigate the orienting performance of BCI-based electrical stimulation in rats with electrodes implanted in the VPM.

One significant finding of our study was the increased glucose metabolism in the paraventricular thalamic nucleus, amygdala, hippocampus, and lateral septum after VPM stimulation. The paraventricular thalamic nucleus is a major source of projections to the nucleus accumbens, the bed nucleus of the stria terminalis, and the central nucleus of the amygdala, as well as to the cortical areas associated with these subcortical regions. The paraventricular thalamic nucleus participates in functional integration of limbic cortical and striatal circuitry and is notable for providing a very dense projection to the nucleus accumbens, a part of the striatum strongly associated with regulation of locomotion (10). Consequently, the paraventricular thalamic nucleus, with its direct and indirect projections to the nucleus accumbens, forms an impressive neural network that can exert control over locomotor activity (11). The amygdala is a critical component of the neural circuitry underlying fear associations and emotional learning (12). Previous work indicated an essential role for the basolateral amygdala in stimulus-reward learning and for the dorsal hippocampus in spatial learning and memory (13). Interestingly, the current study found increased glucose metabolism not only in the amygdala and 


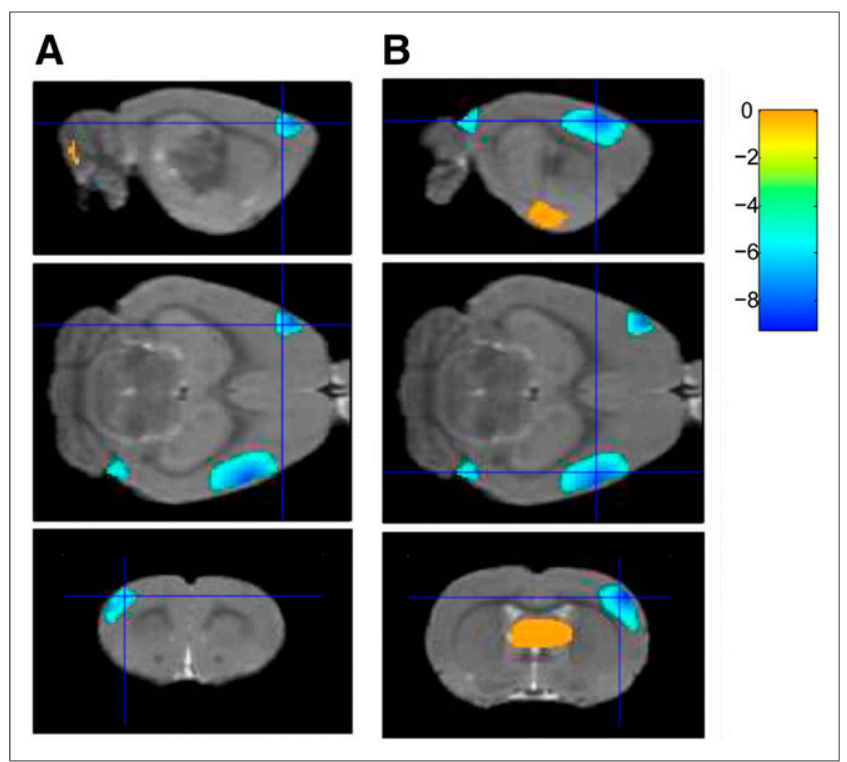

FIGURE 4. Representative sagittal (top), transverse (middle), and coronal (bottom) PET images demonstrating decreased glucose metabolism in left $(A)$ and right $(B)$ primary somatosensory cortices $(P<0.001)$ after VPM stimulation.

paraventricular thalamic nucleus but also in the septohippocampal nucleus and bilaterally in the lateral septumobservations that are consistent with recent literature on the hippocampus-to-lateral-septum pathway (14). The optogenetic stimulation of projections from the lateral septum to the lateral hypothalamus decreased locomotion, suggesting that the lateral septum has a crucial role in controlling locomotion and arousal.

The insular cortex, a part of the neocortex located in the lateral temporal lobe, is highly interconnected with multiple brain networks (15). Electrical stimulation of the insula has been shown to elicit body movements in nonhuman primates, indicating a role in sensorimotor processing (16). The midposterior insula has also been implicated in the processing of awareness of the position, movement, and sensation of the body and thus has a role in coordinating movements effectively (17). The cerebellum has been implicated in the control of actions and the acquisition of motor skills (18). In particular, the posterolateral regions (crus I and II) have been shown to be involved in adaptations to force fields and to visuomotor perturbation (19). Taking these findings from the literature and our findings together, we suggest that the insula and cerebellum may be involved in coordination of movement and control of the turning angle.

Our finding that electrical stimulation of the VPM decreases glucose metabolism in the primary somatosensory cortex is supported by other studies. Electrical stimulation has been shown to evoke strong and long-lasting cortical inhibition that suppresses firing of neurons (20). A recent study investigating responses in the visual cortex (21) found that visual stimuli evoke fewer spikes in the awake state than in the anesthetized state and that spikes evoked in the awake state are quickly followed by a significant and long-lasting hyperpolarization, suggesting that cortical responses to sensory stimulation are dominated by synaptic inhibition in the awake cortex (21). Thus, we speculate that electrical stimulation of the VPM might lead to initial activation of the cortical pyramidal neurons; subsequently, thalamocortical input recruited by strong postsynaptic inhibition shunts the initial excitatory input within the course of the hyperpolarization, resulting in a long-lasting decrease in baseline activity of the primary somatosensory cortex. Greater insight into the effect of BCI-based stimulation on brain function may be gained by future PET studies that combine electrophysiologic recordings and electroencephalography in the same subject.

\section{CONCLUSION}

This report has described several important findings on the role of specific brain regions involved in VPM stimulation. The study demonstrated that PET mapping after VPM stimulation can identify specific brain regions associated with orienting performance. PET molecular imaging may be an important approach for BCI-based research and its clinical applications. Future studies of interregional neural connectivity using multimodality imaging are needed to provide more insight into BCIbased stimulation.

\section{DISCLOSURE}

The costs of publication of this article were defrayed in part by the payment of page charges. Therefore, and solely to indicate this fact, this article is hereby marked "advertisement" in accordance with 18 USC section 1734. This research was supported by grant 2013CB329506 from the National Key Basic Research Program of China, grant LR13H180001 from the Zhejiang Provincial Natural Science Foundation of China, grants 81425015, 81271601, and 61305145 from the National Natural Science Foundation of China, and grants 20130101110015 and 20130101120166 from the Specialized Research Fund for the Doctoral Program of Higher Education. No potential conflict of interest relevant to this article was reported.

\section{ACKNOWLEDGMENT}

We thank Chaonan Yu for technical support with animal surgery.

\section{REFERENCES}

1. O'Doherty JE, Lebedev MA, Ifft PJ, et al. Active tactile exploration using a brain-machine-brain interface. Nature. 2011;479:228-231.

2. Fitzsimmons NA, Drake W, Hanson TL, Lebedev MA, Nicolelis MA. Primate reaching cued by multichannel spatiotemporal cortical microstimulation. J Neurosci. 2007;27:5593-5602.

3. Talwar SK, Xu S, Hawley ES, Weiss SA, Moxon KA, Chapin JK. Rat navigation guided by remote control. Nature. 2002;417:37-38.

4. Xu K, Zhang J, Zhou H, Lee JC, Zheng X. A novel turning behavior control method for rat-robot through the stimulation of ventral posteromedial thalamic nucleus. Behav Brain Res. 2016;298:150-157.

5. Petersen CC. The functional organization of the barrel cortex. Neuron. 2007;56: 339-355.

6. Watson C, Paxinos G. The Rat Brain in Stereotaxic Coordinates. New York, NY: Elsevier Inc.; 2007. 
7. Casanova JP, Madrid C, Contreras M, Rodriguez M, Vasquez M, Torrealba F. A role for the interoceptive insular cortex in the consolidation of learned fear. Behav Brain Res. 2016;296:70-77.

8. Chapin JK, Moxon KA, Markowitz RS, Nicolelis MA. Real-time control of a robot arm using simultaneously recorded neurons in the motor cortex. Nat Neurosci. 1999;2:664-670.

9. Min BK, Marzelli MJ, Yoo SS. Neuroimaging-based approaches in the braincomputer interface. Trends Biotechnol. 2010;28:552-560.

10. Li Y, Li S, Wei C, Wang H, Sui N, Kirouac GJ. Orexins in the paraventricular nucleus of the thalamus mediate anxiety-like responses in rats. Psychopharmacology (Berlin). 2010;212:251-265.

11. Li Y, Li S, Sui N, Kirouac GJ. Orexin-A acts on the paraventricular nucleus of the midline thalamus to inhibit locomotor activity in rats. Pharmacol Biochem Behav. 2009;93:506-514.

12. Roozendaal B, McEwen BS, Chattarji S. Stress, memory and the amygdala. Nat Rev Neurosci. 2009;10:423-433.

13. McDonald RJ, Yim TT, Lehmann H, et al. Expression of a conditioned place preference or spatial navigation task following muscimol-induced inactivations of the amygdala or dorsal hippocampus: a double dissociation in the retrograde direction. Brain Res Bull. 2010;83:29-37.
14. Bender F, Gorbati M, Cadavieco MC, et al. Theta oscillations regulate the speed of locomotion via a hippocampus to lateral septum pathway. Nat Commun. 2015;6:8521.

15. Kurth F, Zilles K, Fox PT, Laird AR, Eickhoff SB. A link between the systems: functional differentiation and integration within the human insula revealed by meta-analysis. Brain Struct Funct. 2010;214:519-534.

16. Showers MJ, Lauer EW. Somatovisceral motor patterns in the insula. J Comp Neurol. 1961;117:107-115.

17. Christopher L, Koshimori Y, Lang AE, Criaud M, Strafella AP. Uncovering the role of the insula in non-motor symptoms of Parkinson's disease. Brain. 2014;137: 2143-2154.

18. Manni E, Petrosini L. A century of cerebellar somatotopy: a debated representation. Nat Rev Neurosci. 2004;5:241-249.

19. Donchin O, Rabe K, Diedrichsen J, et al. Cerebellar regions involved in adaptation to force field and visuomotor perturbation. J Neurophysiol. 2012;107: $134-147$.

20. Logothetis NK, Augath M, Murayama Y, et al. The effects of electrical microstimulation on cortical signal propagation. Nat Neurosci. 2010;13:1283-1291.

21. Haider B, Hausser M, Carandini M. Inhibition dominates sensory responses in the awake cortex. Nature. 2013;493:97-100. 